\title{
Energy-Efficient On-Chip Networks through Profiled Hybrid Switching
}

\author{
Yuan He \\ isaacyhe@acm.org \\ Shenyang University of Technology, Liaoning, China \\ The University of Tokyo, Tokyo, Japan \\ Thang Cao \\ cao@mitech.jp \\ MITECH Corporation \\ Tokyo, Japan
}

\author{
Jinyu Jiao \\ jiaojinyu@acm.org \\ Shenyang University of Technology \\ Liaoning, China \\ Masaaki Kondo \\ kondo@hal.ipc.i.u-tokyo.ac.jp \\ The University of Tokyo, Tokyo, Japan \\ RIKEN, Kobe, Japan
}

\begin{abstract}
Virtual channel (VC) flow control is the de facto choice for modern networks-on-chip (NoCs) to allow better utilization of the link bandwidth through buffering and packet switching (PS), which are also the sources of large power footprint and long per-hop latency. However, bandwidth can be plentiful for parallel workloads under VC flow control. Thus, dated but simpler mechanisms, such as circuit switching (CS), can help improve the energy efficiency of modern NoCs. In this paper, we propose to apply CS to part of the link bandwidth so that a considerable amount of traffic can be transmitted bufferlessly without routing. Evaluations reveal that this proposal leads to a reduction of energy per flit by up to $32 \%$ while also provides very competitive latency when compared to networks under VC flow control.
\end{abstract}

\section{CCS CONCEPTS}

- Networks $\rightarrow$ Network on chip; • Computer systems organization $\rightarrow$ Interconnection architectures; Multicore architectures. KEYWORDS

networks-on-chip, circuit-switching, virtual channels, latency, energy

\section{ACM Reference Format:}

Yuan He, Jinyu Jiao, Thang Cao, and Masaaki Kondo. 2020. Energy-Efficient On-Chip Networks through Profiled Hybrid Switching. In Proceedings of the Great Lakes Symposium on VLSI 2020 (GLSVLSI '20), September 7-9, 2020, Virtual Event, China. ACM, New York, NY, USA, 6 pages. https://doi.org/10. $1145 / 3386263.3406934$

\section{INTRODUCTION}

With increasing number of cores on die, the demand for scalable and efficient on-chip networks is persistent. To meet the stringent performance requirement, VC flow control has long been employed

Permission to make digital or hard copies of all or part of this work for personal or classroom use is granted without fee provided that copies are not made or distributed for profit or commercial advantage and that copies bear this notice and the full citation on the first page. Copyrights for components of this work owned by others than the author(s) must be honored. Abstracting with credit is permitted. To copy otherwise, or republish, to post on servers or to redistribute to lists, requires prior specific permission and/or a fee. Request permissions from permissions@acm.org.

GLSVLSI '20, September 7-9, 2020, Virtual Event, China

(C) 2020 Copyright held by the owner/author(s). Publication rights licensed to ACM. ACM ISBN 978-1-4503-7944-1/20/09...\$15.00

https://doi.org/10.1145/3386263.3406934 by mainstream NoC designs for better utilization of link bandwidth through buffering and arbitrations. However, it increases both power consumption and per-hop latency significantly due to the following reasons. First, flit buffers are a major source of static power in NoCs. Secondly, accesses to these buffers can draw a significant amount of dynamic power. Thirdly, routing at each hop and buffer allocations/accesses deepen the router pipelines and further increase the latency. To tackle these concerns, many optimization techniques are proposed [2, 5, 7, 9, 11, 13, 19-22]. Despite the effectiveness of such techniques directly addressing power and performance issues, there are also attempts that question the necessity of VC flow control, buffering and arbitrations in modern NoCs. For example, hybrid switching (HS) which employs both modern and dated flow controls (such as CS) in the same network may bring sophistication and efficiency at the same time [6, 14, 15, 18, 23].

On one hand, CS is a flow control designed for simplicity. It approaches wire delay and realizes bufferless operations. However, such simplicity also limits throughput as setting up circuits requires longer time than the actual traversal of flits. In the process of circuit set-up, all links on a circuit have to be reserved and such reservations have to be acknowledged at the source where traffic is sent. Therefore, contentions may easily occur as the route occupied by a packet cannot be shared with a different packet. On the other hand, VC flow control is employed to allow higher link utilization and throughput with more advanced but power hungry designs (such as buffering and PS). Obviously, applying HS may bring advantages from multiple flow control mechanisms.

Existing studies $[6,14,15,18,23]$ on HS were mainly set out for when and where circuits are formed. One way is to vary the link status and circuit set-up over time so that a particular link may be included in different circuits or simply used for PS at different time to fit the traffic (time division multiplexing, or TDM for short). The other way is to set up circuits with particular amount of link bandwidth so that multiple circuits can be formed across the same link (space division multiplexing, or SDM for short). Moreover, there are also proposals which combine both TDM and SDM to allow more efficient circuit set-up and usage.

Nevertheless, for above studies, circuit set-up is carried out in a per-packet manner. Therefore, in this work, we attempt to both simplify the circuit set-up process and to alleviate its overheads, such as the set-up delay, from previous works with the help of traffic regularity. We separate the network into multiple logical 
subnets so that one of them is always under VC for correctness of operation while all other logical subnets are under CS but the circuits are formed according to past traffic patterns. In more details, circuit set-up is carried out for CS subnets with highly-repeated traffic patterns (traffic regularity) from profiling as we find that for most of the application workloads, traffic can be very regular. Hence, frequently traversed routes are therefore set as circuits in the CS subnets so that traffic traveling through such routes is always under the CS flow control. On the other hand, traffic which cannot be transmitted through CS subnets will be transmitted in the VC subnet to guarantee the functioning of the network. This proposal also brings a novel way of allocating the link bandwidth, that is, most of it can be dedicated to bufferless circuits as long as a small share of it is buffered to avoid reforming the circuits.

Obviously, the key to our proposal is to ensure the formed circuits can host as much traffic as possible. To efficiently and effectively form the circuits, we have proposed two circuit set-up algorithms. The first algorithm is "greedy". With this algorithm, we search through the traffic trace to find the most frequentlytraversed paths and use them as candidates for circuit set-up. With the second one, we try to maximize circuit traversals with a "genetic algorithm (GA)". Both algorithms are utilized under two different situations, 1) offline static circuit set-up and 2) adaptive circuit setup at runtime. Although "GA" can be more effective than "greedy", it is also more heavy-weight therefore impractical for runtime usage.

With this proposal and our two algorithms, we can improve the energy efficiency of on-chip networks for the following reasons. First, dynamic and static power of the network can be reduced when traffic traverses the network through CS subnets. Second, performance may also be improved since per-hop latency is shortened for such traffic. Third, this proposal can be built on existing modern NoC designs with very small modifications to the router. Fourth, both the static approach and the runtime adaptive approach allow us to effectively capture the traffic regularity.

The main contributions of this paper are summarized as follows.

- We confirm an important fact that traffic in a network has regularity so that most of the traffic can benefit from circuitswitched designs without frequently reforming circuits.

- We present effective approaches to form circuits statically or adaptively in CS subnets after/at runtime and they only require slight modifications to state-of-the-art router designs.

- We reveal latency and energy trade-offs among the number of CS subnets, the way of circuit set-up and the amount of traffic in circuits for different applications. This further helps determining the best solution for the on-chip bandwidth allocation problem.

The rest of this paper is organized as follows. Section 2 covers drawbacks of the VC flow control in modern NoCs and motivates our work, while Section 3 presents our proposal. In Section 4, we cover the evaluation methodology. Section 5 then presents our results and discussions. Section 6 introduces the related work and Section 7 concludes this paper.

\section{BACKGROUND AND MOTIVATIONS}

From CS to VC, NoCs evolve for better throughput by having more and more advanced designs such as buffering and deeper

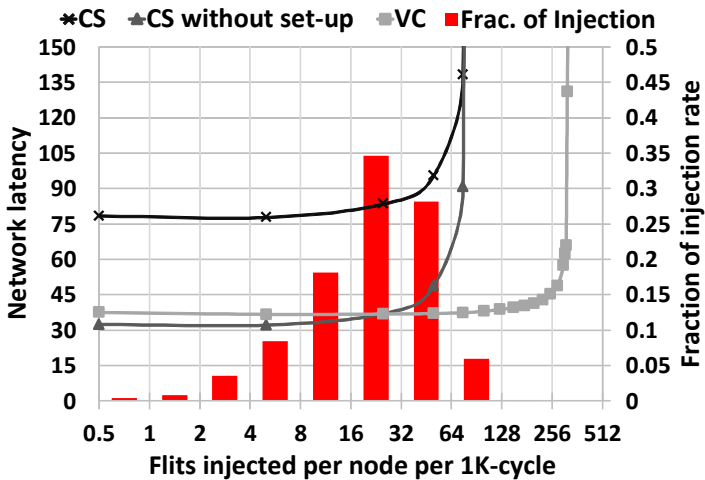

Figure 1: Latency versus injection for different flow controls and the injection rate of an application workload.

pipelining. Both dynamic and static power consumptions are increasing as more advanced flow controls are employed. Hop latency is also getting larger since more advanced flow controls involve buffer accesses and more complex resource allocations. As can be seen from Fig. 1, CS saturates more quickly than VC and it also suffers from the circuit set-up delay. But when circuit set-up delay is excluded and the network is not busy, CS may provide enough throughput while retaining lower latency. It also can be seen from Fig. 1 with injection rate changes of an application that most of the time, an application does not inject much into the network. On the other hand, it is no doubt that VC flow control excels in providing great throughput. However, with the end of Dennard scaling, power consumption and energy efficiency are becoming more and more important metrics when designing a system. This also makes the power consumption of NoCs a more critical issue and is the reason why simpler flow controls need to be re-considered.

Another important fact is, traffic in an on-chip network can repeat. For example, in the system evaluated in this work, the network has 51 network interfaces so that there are in total 2550 possible paths in theory. On the other hand, for each one of the eight benchmark programs we tested, there are around 60 to 110 million flits of network traffic traveling in such 2550 possible paths. In practice, some paths (from some particular cores to some particular banks of cache and vice versa) may simply have more traffic traversing through them than through others. Such traffic regularity means, if we set up circuits for most frequently traversed paths, we can have large amount of traffic traversing them. Moreover, if we further split the runtime of an application into epochs, we can adapt to regularity changes from time to time.

\section{HYBRID SWITCHING AND LINK WIDTH ALLOCATIONS}

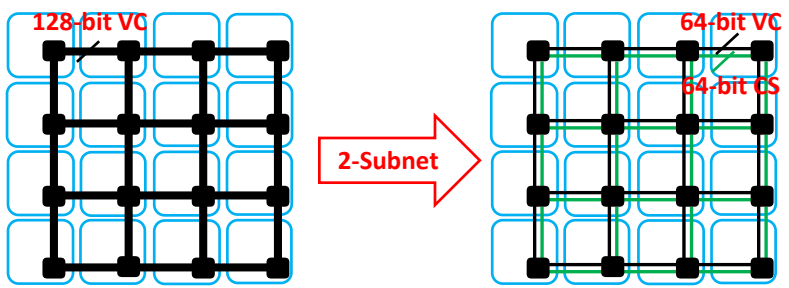

Figure 2: Physical links (under VC flow control) divided into two subnets (one under VC while the other under CS). 


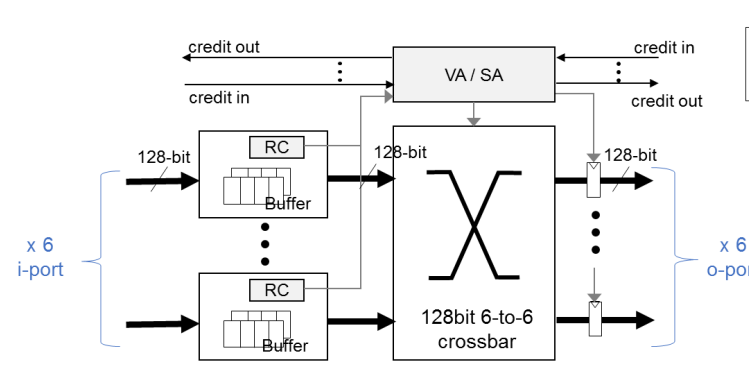

(a) Conventional router design.

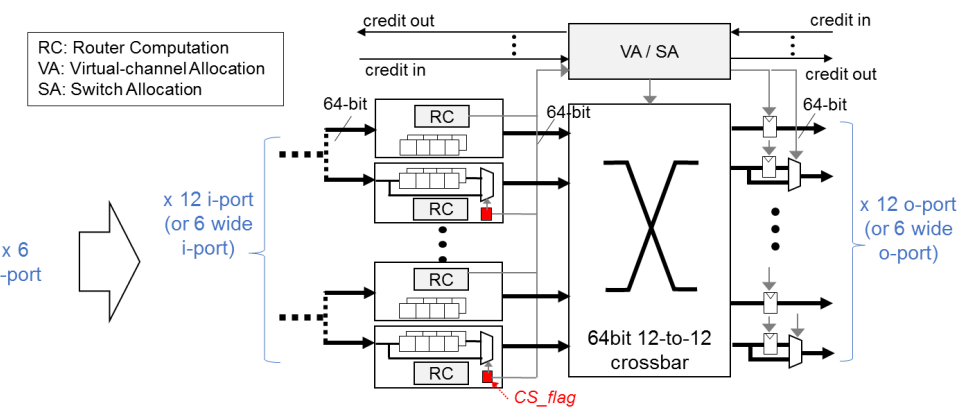

(b) Reference hybrid-switched router design.

Figure 3: Router micro-architecture and extensions for the hybrid flow control with CS and VC.

\subsection{Enabling CS and VC Flow Controls in a Single Network}

To enable both CS and VC flow controls in a network, we employ the idea of SDM [15] so that links in the network are partitioned into different subnets and different flow controls (such as VC and CS) are applied. For example, if the link bandwidth of the network is 128-bit, we can divide the physical datapath into a VC subnet and a CS subnet so that each one is 64-bit wide as in Fig. 2. For more circuits to be formed, we can further divide the network into more subnets which is equal to having more CS planes.

HS with SDM needs modifications to the router as illustrated in Fig. 3. We assume the conventional router has six input/output ports and the link width is 128-bit. When the physical links are divided into two subnets. The HS router then has twelve input/output ports so that each of them has a 64-bit link width. There is a one-bit flag (CS_flag) associated with each input port of the potential CS subnet specifying whether the corresponding port is utilized to form a circuit. If the flag is set, an input flit is directly sent to the crossbar. The flag also goes to one of the output ports through the VA/SA unit to help fix the path in the crossbar switch and bypass the latch on the output port. Hence, a flit coming to the input port immediately travels to the next hop. One of the drawbacks of this design is that the complexity of the crossbar slightly increases.

Since CS transfers data in a pre-formed path, only injected flits whose source and destination match with one of the formed circuits can take advantage of the CS flow control. There can be multiple circuits simultaneously unless they compete with each other for an input/output port of a router or a link between routers. Nevertheless, it is impossible to set up circuits for all combinations of sources and destinations at the same time. Thus, traffic which cannot use CS subnet should go through the VC subnet. In the conventional CS flow control, once a new packet is injected into the network and there is no circuit for it, it first creates a new circuit and then starts the data transfer. This incurs long latency and hinders other packets from using the corresponding links or ports of the routers on the formed circuit. Therefore, our proposal is free from above drawbacks of the conventional CS flow control.

In general, traffic transferred with CS flow control needs neither buffering nor arbitrations within a router, so we expect a shorter transmission delay and reduced power consumption for such traffic. To take these advantages, it is preferable to have more flits in circuits. Therefore, to form more circuits, we further divide the physical datapath into more subnets, which allows more circuits.
However, such a division further shrinks the width of each subnet. This results in higher propagation delay. For the transmission delay, when a flit travels in a circuit, we assume immediate traversal through the router ( 1 cycle in a router), so if a flit travels 3 hops in a circuit, it requires 7 cycles in total to reach the destination (1 cycle per router and 1 cycle per link). In the router design of our proposal (Fig. 3), buffers are still kept but when their associated subnets are configured to circuits, they are simply power-gated.

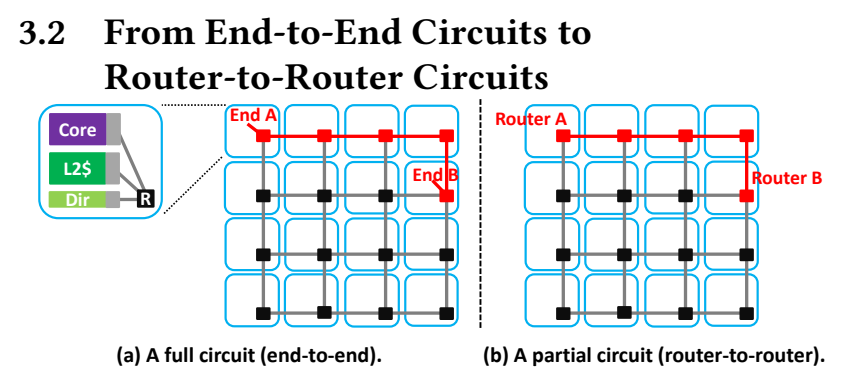

Figure 4: Typical examples of full/partial circuits.

For a typical 16-core chip-multiprocessor (CMP) system in our evaluations, there are in total 51 network interfaces (16 cores, 16 banks of L2 cache, 16 directory controllers, 2 DMA controllers and 1 $\mathrm{I} / \mathrm{O}$ controller). Forming circuits for 51 sources and destinations is a hard task where network links may run out easily. It is obvious that with our proposal, the more circuits can be formed, the better we can potentially improve both network latency and energy efficiency.

To allow more traffic in circuits, we decide to set a relaxation policy that circuits are partial from router to router rather than from end to end (as in Fig. 4). To enable such a relaxation, we need to retain the routing and arbitration at the first and last routers for any circuit traversal. This means, the routing and arbitration latency at such routers still exists. For example, a flit travels 3 hops in this case will only have CS traversal at the second hop. In some cases, this may be beneficial since such a penalty can be alleviated by having more flits in circuits.

\subsection{Approaches for Circuit Set-Up}

In this subsection, we propose to set up the circuits in two approaches, static and runtime adaptive.

3.3.1 Static. Traffic profiles of applications need to be collected before production runs. Circuits can then be formed offline. The advantage is that the application will not be affected when it is executed. And circuit set-up can also be done sufficiently with 
advanced algorithms. The problem with this approach is, it requires profiling and an application has to be executed to collect its traffic profile before this approach can be applied.

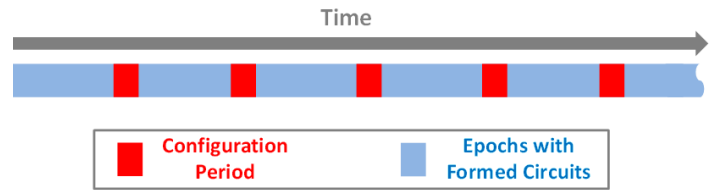

Figure 5: Steps to form circuits adaptively at runtime.

3.3.2 Runtime Adaptive. To avoid profiling runs, we also propose an runtime adaptive approach. The runtime of an application is divided into epochs and within an epoch, circuits are set up for nonconflicting paths based on traffic profiles of the previous epoch (as in Fig. 5). So for current epoch, flits matching the source and destination of formed circuits can traverse them. On the other hand, traffic which do not match the source and destination of formed circuits will traverse the network through the VC subnet. This approach is able to catch traffic pattern changes over different phases of runtime. When the traffic profile of an epoch is collected, we assume the circuit set-up process is carried out with the help of a software. Within a configuration period, it will gather stats from network interfaces and routers, carry out circuit set-ups and send circuit configurations back. The length of this configuration period is set to 1 million cycles since our greedy algorithm can finish within this amount of time, but not the genetic algorithm (GA). For this approach, GA is used for comparison only.

\subsection{Algorithms for Circuit Set-Up}

With our proposal, forming circuits is the most important issue but it is not easy even with complete traffic profiles since we are only able to accommodate part of the candidate circuits with high traffic load due to conflicts and this is an NP-hard problem. We thus propose two algorithms to help.

3.4.1 Greedy Algorithm. The first algorithm to form circuits is based on greedy allocation. After collecting traffic stats for each pair of source and destination, they will be sorted in descending order for the total amounts of hops (number of hops $\times$ number of flits) and CS paths will be formed from the top of this list while conflicting CS connections are simply discarded. Since this algorithm prioritizes frequently used source and destination pairs as circuit candidates, it is able to find good combinations of circuits for given traffic patterns. However, one problem with this algorithm is, one chosen circuit may prevent some further candidates to be chosen because of conflicts but these discarded ones may not conflict with each other and can thus provide better potential in accelerating more traffic when chosen together. Therefore, for this optimization problem, greedy algorithm cannot guarantee the best solution.

3.4.2 Genetic Algorithm. As the greedy algorithm does not necessarily generate the best combinations of circuits and the entire search space is too large to be solved, we try to use a well known heuristic solution to find better answers. The second algorithm is based on GA. In our GA formulation, each entry of the chromosomes corresponds to all pairs of sources and destinations and a bit is used to represent if any pair of source and destination is set as a circuit. When being applied to find the solution, we start with
10 solutions we found with the greedy algorithm as the starting 10 individuals for GA and apply crossover ( 0.3 to 0.7 for all individuals) and mutation ( 0.5 for a chromosome) to search for better individuals in 5000 generations. Note that this iterative algorithm cannot be used for online adaptive circuit set-up. This is mainly proposed for offline usage (static) but we also use it when comparing to the online approach (runtime adaptive).

\section{METHODOLOGY}

In this paper, evaluations are carried out with gem5 [4] extended with the network model from GARNET [1] and the power model from McPAT [17]. To evaluate the performance, we have modified the source code of gem5 and GARNET to provide cycle-accurate timing model of our proposal. For the energy evaluation, we simply feed performance statistics collected from gem5 to McPAT.

Table 1: Evaluation parameters.

\begin{tabular}{ll}
\hline Number of cores: & 16 \\
Topology: & $4 \times 4$ mesh \\
Processor: & $2 \mathrm{GHz}$, In-order \\
L1 I/D cache: & 32 KB per Processor, \\
& 4-way set associative \\
L2 cache: & 256 KB per Bank, \\
& 16-way set associative \\
Cache line: & 64 Bytes \\
Main memory: & $8 \mathrm{~GB}$ \\
Main memory latency: & $50 \mathrm{~ns}$ \\
Coherence protocol: & MOESI, Directory \\
Link: & 128 -bit, 1 cycle traversal \\
Packet: & 128 -bit control, 640-bit data \\
Router: & $2 \mathrm{GHz}$, 4-cycle virtual channel router \\
Virtual channel: & 4 per Virtual network \\
Virtual network: & 3 per Physical link \\
Routing algorithm: & X-Y routing \\
Process technology: & 22 nm \\
Vdd: & $1 \mathrm{~V}$ \\
\hline
\end{tabular}

In our evaluations, we assume a 16-tile mesh network with 128bit links. Each node has an in-order processor core, a bank of L2 cache/directory. These components are connected to a router individually (as shown in Fig. 4). More details are shown in Table 1. Our evaluations are conducted with applications from the PARSEC benchmark suite [3] with the input size of "simlarge". Due to page limitation, we only present results with the length of epochs set to 200 million cycles. When comparing latency and energy per fit, results obtained with our proposal are normalized to the latency and energy per flit under conventional VC.

\section{RESULTS AND DISCUSSIONS}

\subsection{Results under the Static Approach}

The first set of results we present in Fig. 6 is the percentage of flits in circuits, normalized latency and energy per flit for various workloads under the static approach when circuits are end-to-end. It can be seen that our proposal is very effective. When having 8 subnets, up to $40 \%$ of the flits can traverse the network through endto-end circuits and this results in a reduction of energy per flit for up to $32 \%$ with only a slight increase of latency per flit (about $1 \%$ ). When having 4 subnets, we can observe a per-flit latency reduction of about $8 \%$ while also suppressing the energy for up to $23 \%$ per flit. 

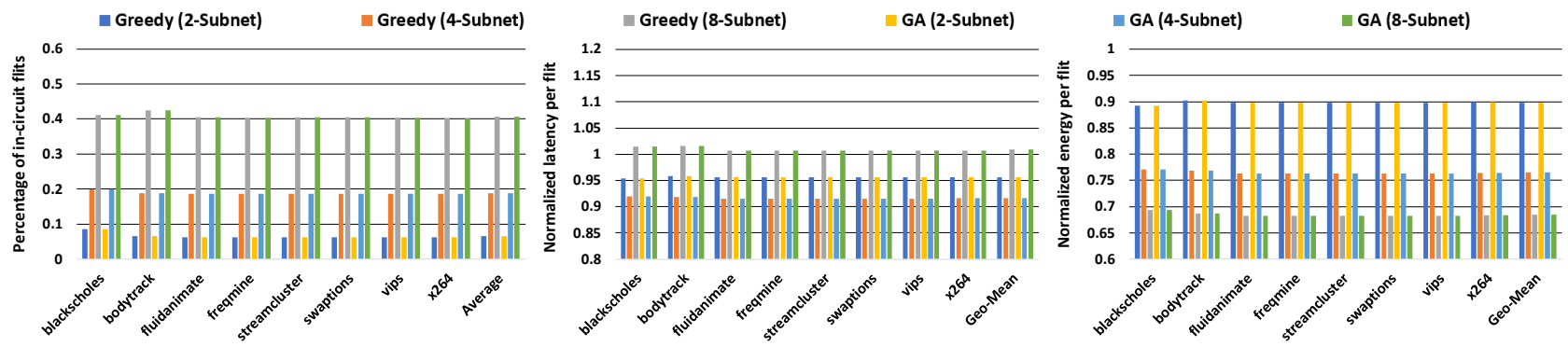

Figure 6: Evaluation results under the static approach with end-to-end circuits.
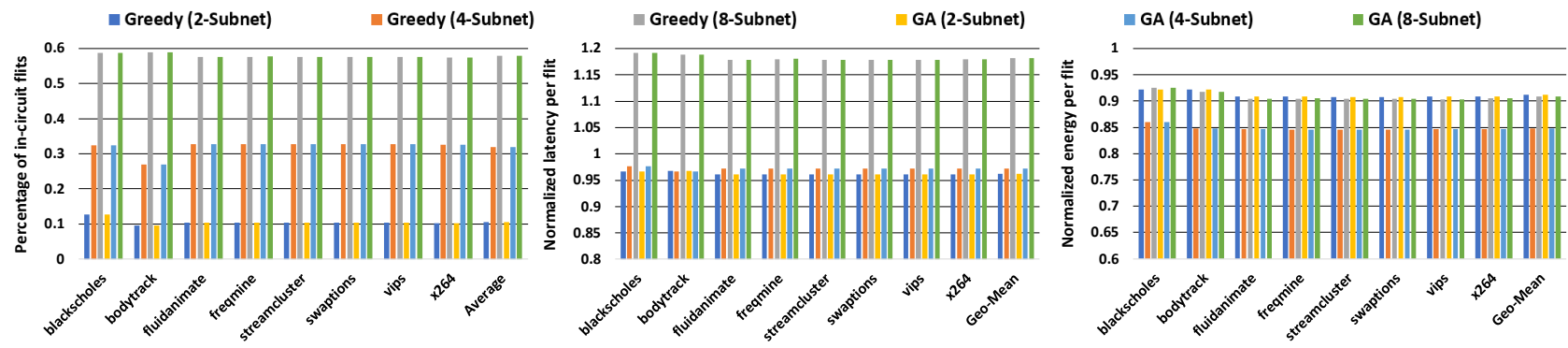

Figure 7: Evaluation results under the static approach with router-to-router circuits.
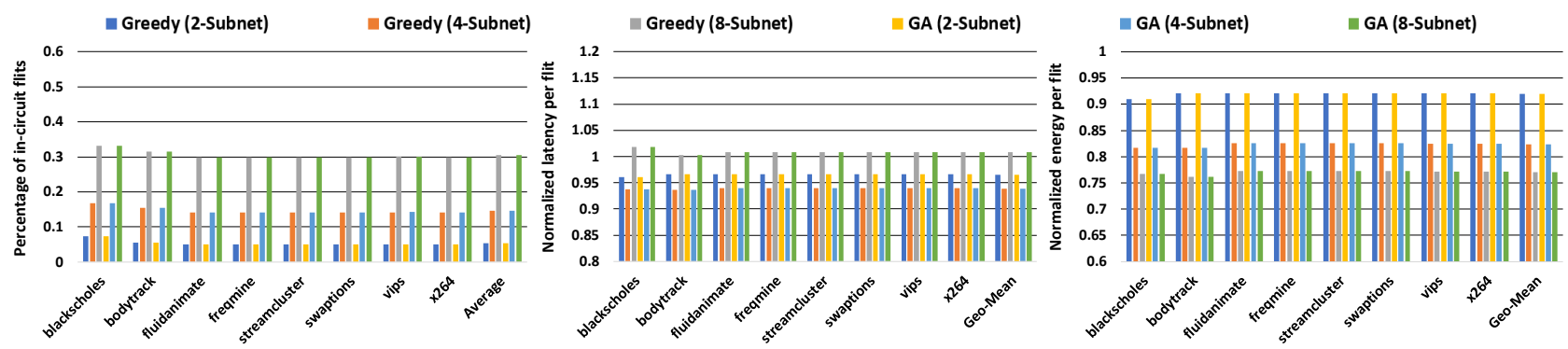

Figure 8: Evaluation results under the adaptive approach with end-to-end circuits.
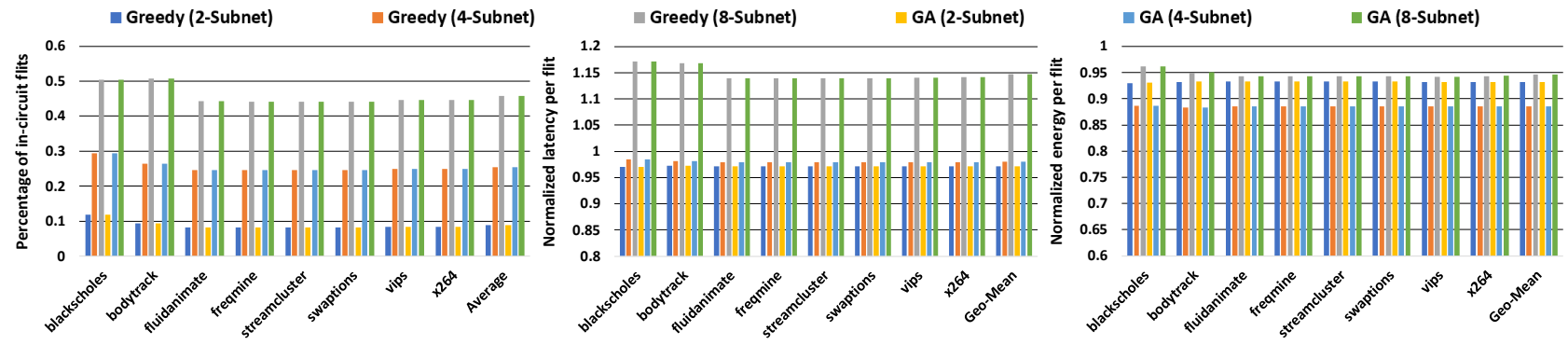

Figure 9: Evaluation results under the adaptive approach with router-to-router circuits.

Moreover, when circuits are formed with the router-to-router relaxation (as in Fig. 7), we can see that nearly $60 \%$ of the flits can traverse the network through circuits under 8 subnets. However, this large increase in in-circuit traffic is not well reflected in both latency and energy per flit as flits still need routing and buffering at its first and last hops when traversing router-to-router circuits. With this relaxation, we observe the best latency reduction per flit under 2 subnets for up to $4 \%$ and the best energy reduction per flit under 4 subnets for about 15\%. One further observation from Fig. 6 and Fig. 7 is, for the static approach, the greedy algorithm works as good as GA.

\subsection{Results under the Adaptive Approach}

For the adaptive approach, from Fig. 8, we can see the percentage of flits in circuits, normalized latency and energy per flit for various workloads when circuits are end-to-end. Our proposal is still very effective at runtime. When having 8 subnets, up to $30 \%$ of the flits can traverse the network through end-to-end circuits and this results in a reduction of energy per flit for up to $23 \%$ with only a slight increase of latency per flit at about $1 \%$. On the other hand, when having 4 subnets, we can observe a per-flit latency reduction of about $6 \%$ while also lowering the energy for up to $17 \%$ per flit.

Moreover, when circuits are formed with the router-to-router relaxation at runtime (as in Fig. 9), we can see that up to $50 \%$ (for 
"blackscholes" and "bodytrack") of the flits can traverse the network through the circuits. Again, this large increase in in-circuit traffic is not well reflected in both latency and energy per flit as flits need routing and buffering at its first and last hops when traveling in router-to-router circuits. With router-to-router circuits under our adaptive approach, we see the best latency reduction per flit comes at 2 subnets for up to $3 \%$ and the best energy reduction per flit with 4 subnets for about $11 \%$.

Although this adaptive approach is not as good as the static one in either latency or energy reduction, it is more useful as no advanced profiling before the production run is needed. The profiling and circuit set-up are carried out online at runtime with this approach. One similar observation from Fig. 8 and Fig. 9 to results under the static approach is, no obvious advantage can be seen for the genetic algorithm. This is a good news as "GA" is impractical for this runtime adaptive approach.

Last but not least, when looking at both static and adaptive results with two types of circuits, we can observe energy reductions over VC in all cases. This means, although the impact of our proposal on latency is not always positive, it is promising for energy.

\section{RELATED WORK}

Energy efficiency is one of the most critical metrics for computer system design but its importance has seen a dramatic leap since dark silicon phenomenon was forecast [10]. Our work simply builds from this valuable insight since we believe NoC is not something to overlook. Their performance and power impacts are worth looking into to further improve the energy efficiency of the system.

So far, many NoC optimization techniques have been studied in order to shrink the power consumption of NoCs. There are many existing works on saving the static power of routers through power management techniques such as power gating [19], suppressing the static power through shared buffer design [11], proportionally supplying power to the network based on traffic demand [8] or completely eliminating routers through smart wiring techniques [2]. These optimization techniques can be very useful towards their purposes and they tried to re-balance power and performance for NoCs, but there is no such work which tries to look at energy efficiency from the viewpoint of flow controls of NoCs yet.

On the other hand, there are many existing works focusing on shortening the latency of routers [9,12,13, 16, 20-22]. Additionally, Kumar et al. proposed express channels which enable multi-hop packets to bypass intermediate routers [16]. Although this work has similar motivations to ours, its hardware overheads is more significant due to credit management.

Networks mixing CS and VC flow controls are started from [15] and the interconnection fabric was shared by different flow controls based on SDM. It is followed by $[6,14,23]$ while these three rely on TDM instead. Furthermore, both SDM and TDM are employed in [18] in order to promote the utilization of circuits. Although both [15] and our work are based on SDM, ours differs very much from it in the way of setting up circuits. Jerger et al. uses a lightweight but separate network to set up circuits for all packets individually while ours focuses on setting up circuits for some of the packets through profiling for the whole execution period or within a certain epoch and such set-up is refreshed in every epoch.

\section{CONCLUSIONS}

Flow control is an important aspect of NoCs since it determines how traffic is treated and is highly related to both performance and energy consumption of the network. In this paper, we proposed a novel NoC design which has its datapath divided into several independent subnets so that some of them can be operated under CS flow control in order to lower the per-hop latency and energy consumption. We found that this idea of partitioning is able to help reduce the energy consumption of a flit by up to $32 \%$ while shorten its latency by up to $8 \%$. This is due to the elimination of routing and having less accesses to the buffers and also being able to gate them in the CS subnets. Such effectiveness proves that our proposal is more future proof as energy efficiency is more and more important.

\section{ACKNOWLEDGMENTS}

First and foremost, we would like to sincerely thank the anonymous reviewers for their valuable comments. This work was supported, in part, by JST CREST from Japan with Grant JPMJCR18K1 and by the Natural Science Foundation of Liaoning Province in China under Grant 20180550194.

\section{REFERENCES}

[1] N. Agarwal et al. 2009. GARNET: a detailed on-chip network model inside a full-system simulator. In Proc. of ISPASS'09. 33-42.

[2] F. Alazemi et al. 2018. Routerless Network-on-Chip. In Proc. of the 24th HPCA. 492-503.

[3] C. Bienia. 2011. Benchmarking Modern Multiprocessors. Ph.D. Dissertation. Princeton University.

[4] N. Binkert et al. 2011. The Gem5 Simulator. SIGARCH CAN 39, 2 (August 2011), $1-7$.

[5] X. Chen et al. 2012. In-network Monitoring and Control Policy for DVFS of CMP Networks-on-Chip and Last Level Caches. In Proc. of the 6th NoCS. 43-50.

[6] J. Cong et al. 2015. On-chip Interconnection Network for Accelerator-rich Architectures. In Proc. of the 52nd DAC. 8:1-8:6.

[7] R. Das et al. 2008. Performance and Power Optimization Through Data Compression in Network-on-Chip Architectures. In Proc. of the 14th HPCA. 215-225.

[8] R. Das et al. 2013. Catnap: Energy Proportional Multiple Network-on-chip. In Proc. of the 40th ISCA. 320-331.

[9] A. Ejaz et al. 2018. FreewayNoC: A DDR NoC with Pipeline Bypassing. In Proc. of the 12th NoCS. 1-8.

[10] H. Esmaeilzadeh et al. 2011. Dark Silicon and the End of Multicore Scaling. In Proc. of the 38th ISCA. 365-376.

[11] H. Farrokhbakht et al. 2019. UBERNoC: Unified Buffer Power-Efficient Router for Network-on-Chip. In Proc. of the 13th NoCS. 1-8.

[12] M. Hayenga et al. 2011. The NoX router. In Proc. of the 44th MICRO. 36-46.

[13] Y. He et al. 2013. McRouter: Multicast Within a Router for High Performance Network-on-chips. In Proc. of the 22nd PACT. 319-330.

[14] Y. He et al. 2016. Opportunistic Circuit-Switching for Energy Efficient On-Chip Networks. In Proc. of the 24th VLSI-SoC. 1-6.

[15] N.D.E. Jerger et al. 2008. Circuit-Switched Coherence. In Proc. of the 2nd NoCS. 193-202.

[16] A. Kumar et al. 2007. Express virtual channels: towards the ideal interconnection fabric. In Proc. of the 34th ISCA. 150-161.

[17] S. Li et al. 2009. McPAT: An integrated power, area, and timing modeling framework for multicore and manycore architectures. In Proc. of the 42nd MICRO. 469-480.

[18] A.K. Lusala et al. 2012. Combining SDM-based circuit switching with packet switching in a router for on-chip networks. International fournal of Reconfigurable Computing 2012 (September 2012).

[19] H. Matsutani et al. 2010. Ultra Fine-Grained Run-Time Power Gating of On-chip Routers for CMPs. In Proc. of the 4th NoCS. 61-68.

[20] H. Matsutani et al. 2011. Prediction Router: a low-latency on-chip router architecture with multiple predictors. IEEE TC 60, 6 (June 2011), 783-799.

[21] R. Mullins et al. 2004. Low-latency virtual-channel routers for on-chip networks. In Proc. of the 31st ISCA. 188-197.

[22] L.S. Peh et al. 2001. A Delay Model and Speculative Architecture for Pipelined Routers. In Proc. of the 7th HPCA. 255-266.

[23] J. Yin et al. 2014. Energy-Efficient Time-Division Multiplexed Hybrid-Switched NoC for Heterogeneous Multicore Systems. In Proc. of the 28th IPDPS. 293-303. 